\title{
Comunalidades entre las narrativas históricas de estudiantes y textos escolares: un análisis cualitativo
}

Communalities between the Historical Narratives of Students and Textbooks: A Qualitative Analysis

Comunalidades entre as narrativas históricas de estudantes e textos escolares: uma análise qualitativa

\section{Claudia Patricia Navarro-Roldán* (D) Javier Alejandro Corredor-Aristizábal ${ }^{* *}$ (iD orcid.org/0000-0003-0893-6332}

\footnotetext{
Artículo de investigación

Revista Colombiana de Educación, N.7 75. Segundo semestre de 2018, Bogotá, Colombia.

doi: 10.17227/rce.num75-8104

Para citar este artículo: Navarro-Roldán, C. y Corredor-Aristizábal. (2018). Comunalidades entre las narrativas históricas de estudiantes y textos escolares: un análisis cualitativo. Revista Colombiana de Educación, 75, 119-137.
}

$\begin{array}{ll}\text { (c) } \bigcup_{\mathbf{B Y}} \$ \mathbf{N C} & \text { Recibido: 07-02-2017 } \\ & \text { Evaluado: 09-01-2018 }\end{array}$

* Doctora en Psicología. Profesora de planta del programa de psicología de la Universidad Pedagógica y Tecnológica, Tunja, Colombia. Correo electrónico: claudia.navarro@uptc.edu.co

** Doctor en Estudios Cognitivos. Profesor asociado a la Universidad Nacional de Colombia, Bogotá, Colombia. Correo electrónico: jacorredora@anal.edu.co 


\section{Resumen}

Este artículo de investigación da cuenta de un estudio cualitativo que usó el análisis de las narrativas para conocer cómo los textos escolares reconstruyen la Independencia de Colombia, y cómo esta reconstrucción se relaciona con las narrativas independentistas de los estudiantes en contextos socioculturales diversos. Se realizaron 21 entrevistas a estudiantes de octavo grado en dos colegios urbanos y dos rurales. Se utilizó un muestreo por conveniencia y el criterio de saturación de las categorías de análisis Se analizaron los textos escolares que los estudiantes usan en sus clases de ciencias sociales. Las narrativas fueron segmentadas y analizadas a partir de tres categorías historiográficas: fuentes históricas, explicación histórica y motor de la historia. Se encontró que la plantilla historiográfica reproducida en los textos para organizar la información y otorgarle significación histórica fue usada por los estudiantes para construir sus propias narraciones históricas; y que coexisten las mismas narrativas independentistas en contextos rurales y urbanos. Se concluyó que los textos reprodujeron una narrativa histórica nacional que estructuralmente no favorece a los estudiantes en la construcción de conocimiento disciplinar sofisticado.

\section{Palabras clave}

texto; explicación; cultura; historia; narración
Keywords

text; explanation; culture; history; narration

\begin{abstract}
This research article reports a qualitative study that used the analysis of narratives to learn how school texts reconstruct Colombia's independence, and how this reconstruction is related to student's independent narratives in diverse socio-cultural contexts. There were 21 interviews with eighth-grade students in two urban and two rural schools. A convenience sampling and the saturation criterion of the analysis categories was used. The textbooks that students use in their social science classes were analyzed. The narratives were segmented and analyzed from three historiographical categories: historical sources, historical explanation and engine of history. It was found that the historiographic template reproduced in the textbooks to organize the information and grant it historical significance was used by the students to construct their own historical narratives; and that the same independence narratives coexist in rural and urban contexts. It was concluded that the texts reproduced a national historical narrative that structurally does not favor students in the construction of sophisticated disciplinary knowledge.
\end{abstract}

\section{Resumo}

Este artigo de pesquisa apresenta um estudo qualitativo que utilizou a análise das narrativas para conhecer como os textos escolares reconstroem a independência da Colômbia e como essa reconstrução está relacionada com as narrativas independentistas dos estudantes em contextos socioculturais diversos. Realizaram-se 21 entrevistas a estudantes de oitavo ano em dois colégios urbanos e duas escolas rurais. Utilizou-se uma amostragem por conveniência e o critério de saturação das categorias de análise. Analisaram-se os textos escolares que os estudantes utilizam em suas aulas de ciências sociais. As narrativas foram segmentadas e analisadas em três categorias historiográficas: fontes históricas, explicação histórica e motor da história. Descobriu-se que o padrão historiográfico reproduzido nos textos para organizar a informação e adjudicar-lhe significação histórica foi utilizada pelos estudantes para construir suas próprias narrações históricas Também, encontrou-se que coexistem as mesmas narrativas independentistas em contextos rurais e urbanos. Como conclusão, os textos produziram uma narrativa histórica nacional que estruturalmente não favorece os estudantes na construção de conhecimento disciplinar sofisticado.

\section{Palavras-chave}

texto; explicação; cultura; história; narração 


\section{Introducción}

Las narrativas históricas son construidas y reproducidas socialmente. Una de las formas de reproducción de dichas narrativas es la escuela (Barton, 2001; Barton y McCully, 2005; Carretero, Castorina y Levinas, 2011), y pasa, particularmente, por los textos escolares (Alridge, 2006). Esto es así porque los textos escolares son obras historiográficas que reconstruyen la historia que debe ser aprendida por los estudiantes. No obstante, aunque las ciencias de la educación y la psicología han analizado ampliamente cómo se construye el conocimiento histórico, los hallazgos han enfatizado más en las formas en que los textos escolares y las personas (profesores y estudiantes) construyen este conocimiento, $y$ han enfatizado menos acerca del uso, recepción y efectos de los textos en el aula, o sobre la transformación de los saberes de los textos por parte de los estudiantes (Fuchs, 2011). Este estudio apunta a identificar cualitativamente cuáles son las similaridades entre las narrativas presentes en los textos escolares, y las producidas por estudiantes en relación con la Independencia de Colombia. De esta forma, en el artículo se busca mostrar que, al menos desde el punto de vista descriptivo, la historia reconstruida por los estudiantes es similar a lo que narran los textos escolares que usan en el aula de clase como soporte para el aprendizaje.

De esta forma, la pregunta transversal a esta investigación fue: ¿cuál es la relación entre el carácter situado de la comprensión histórica y la mediación de las narrativas históricas reproducidas en los textos escolares? En particular, se pretendió conocer cómo los estudiantes y los textos escolares reconstruyeron el pasado, y cómo esta reconstrucción se relacionó con discursos sociales presentes en el espacio cultural en el cual participan (rural/urbano). Para responder esta pregunta, nos basamos en el supuesto de que la actividad humana, incluida la actividad mental, se construye con el uso de los textos escolares como objetos seleccionados y usados dentro de la escuela (Cole, 1996; Wertsch, 2005). Por ello, empíricamente, se rastrearon las similitudes y diferencias entre la estructura historiográfica de las narraciones de los textos escolares y de las narraciones históricas de los estudiantes en dos contextos sociales disimiles.

\section{Los textos escolares y la enseñanza de la historia en Colombia}

Los textos escolares son herramientas culturales que colocan a disposición de los estudiantes las narrativas históricas que ellos deberían aprender en cada contexto escolar particular. En este sentido, los textos escolares actúan como herramientas mediadoras de las narrativas históricas sobre el pasado, y la forma en que los estudiantes elaboran y comprenden dichas 
narrativas. De esta forma, el uso del lenguaje hablado de los estudiantes y el lenguaje escrito de los textos escolares permiten conocer las formas en las que el conocimiento sobre el pasado de la sociedad es reconstruido dentro del contexto escolar. Esto es, las comunalidades entre las narrativas de los textos escolares y los estudiantes no solo nos hablan de cómo estos dos elementos se relacionan, sino también sobre cómo la sociedad en su conjunto concibe y reconstruye su pasado.

En Colombia, el estudio sobre cómo los textos escolares reproducen el conocimiento histórico es reciente, y cobra relevancia en la medida en que estos constituyen un mecanismo de configuración de la memoria oficial que se reproduce en la escuela (Cardoso, 2001). En particular, la investigación sobre las narrativas históricas independentistas, revitalizada en el marco de la celebración del Bicentenario de la Independencia durante 2010, encontró que la Independencia se reproduce a través de una versión histórica única e inequívoca (Acevedo y Samacá, 2012; Rodríguez, S., 2010; Rodríguez, J., 2004; Samacá, 2010), en la cual las intenciones de la clase criolla, o algunos héroes o caudillos, son el motor de la historia independentista. Esta visión, es historiográficamente inadecuada porque desconoce elementos históricos importantes señalados tanto por la literatura normativa en historia (Aron, 1984; Burke, 1993; González y González, 1998; Veyne, 1984), como por la investigación educativa y psicológica (Ashby, 2004; Lee, 2005).

Por un lado, esta visión privilegia las intenciones y las ideas como factores de cambio conceptual, e ignora las estructuras y las características del contexto en la explicación histórica (Burke, 1993; González y González, 1998). Por otro, esta visión ignora el carácter construido de la historia, e invisibiliza el hecho de que dichas narrativas son el producto de la revisión de múltiples fuentes, que en muchos casos presentan visiones contradictorias (Barton, 2001; Plá, 2005; Wineburg, 2001). Además, esta visión no considera a los estudiantes como negociadores activos de los mensajes y los significados de los textos escolares, que responden a las características del contexto social en el cual el aprendizaje tiene lugar (Repoussi y Tutiaux-Guillon, 2010).

En consecuencia, la forma como los textos escolares reproducen la historia escolar sobre la Independencia del país, o cualquier otro acontecimiento histórico, tiene serias implicaciones en cómo los estudiantes construyen su propio conocimiento histórico. Los textos escolares muestran no solo la historia oficial que se debería aprender y reproducir en la escuela, sino que presentan las formas válidas de construir el conocimiento escolar, las cuales parecieran alejarse de los modelos y procedimientos historiográficos expertos. Esta investigación aporta evidencia en esta dirección. En particular, en este trabajo se verá cómo, si bien existen similitudes amplias 
entre las narrativas de los textos escolares y de los estudiantes, también en algunos casos estos producen narrativas que reelaboran o integran las narrativas presentadas por los textos escolares.

\section{Efectos de la cultura y el aprendizaje en las narrativas históricas}

Estudios comparativos han evidenciado diferencias en el aprendizaje de la historia entre estudiantes de contextos socioculturales distintos. En particular, Barton (2001) encontró diferencias entre los estudiantes de la escuela primaria en Irlanda del Norte y Estados Unidos. El autor argumenta que el currículo irlandés, a partir de métodos y textos expositivos, enfatiza en los cambios históricos en la vida material y social de la sociedad. Como resultado, los estudiantes construyen narraciones históricas sobre las funciones sociales de las instituciones (la ley, la escuela) y los procesos de grupo (exigencias de movimientos sociales). Esto hace que los estudiantes irlandeses elaboren narraciones históricas que relacionan los hechos del pasado con temas más amplios propios de su contexto sociocultural (sometimiento o acoso).

Por el contrario, el currículo de la escuela primaria estadounidense enfatiza en la narración de los cambios teleológicos de la sociedad americana. Como resultado, los estudiantes narran la construcción de la nación a partir de las ideas (inventos) de personas en el pasado y eventos particulares (la primacía de la raza blanca). En esta línea, se ha encontrado que los estudiantes norteamericanos construyen narrativas históricas relacionadas con la búsqueda de la libertad, con las guerras y el progreso social y material, y con la identidad nacional (Barton, 2001; Wertsch, 1999; Wineburg, 2001). Es decir, construyen un conocimiento histórico que enfatiza más en las intenciones de los sujetos, y menos en el rol que estos cumplen dentro de las estructuras sociales.

El contraste entre estudiantes irlandeses y estadunidenses señala que las diferencias en las narrativas históricas se producen no por características de desarrollo cognitivo particulares de los estudiantes, sino por las limitaciones y las posibilidades que los currículos particulares ofrecen al estudiante. Las características socio históricas de la sociedad irlandesa llevan a que los currículos escolares de historia en primaria no incluyan héroes o personajes con el fin de evitar conflictos entre el sector educativo, político y religioso del país. En esta línea, los estudiantes de primaria en sus clases de historia analizan procesos sociales (evolución de la moda) desde edades más tempranas (en comparación con Estados Unidos), y esto posibilita que representen los procesos sociales y su la evolución histórica. 
Recurrir a una narrativa producida en una comunidad particular revela cómo los estudiantes se apropian de su entorno social, al mismo tiempo que informa sobre cómo ellos construyen un significado para las narrativas históricas disponibles en el contexto (Barton, 2001). Solamente en ese contexto particular la narración adquiere un sentido para el narrador y para quienes la escuchan, independientemente de si esta incluye o no todos los detalles históricos (VanSledright, 2008). En consecuencia, las particularidades en el aprendizaje de la historia escolar, en diferentes países o grupos de referencia, podrían estar asociadas al tipo de narrativas históricas que se producen en cada país, y a los valores o tradiciones culturales propias de cada cultura (Dan, Todd y Lan, 2010; Epstein, 2006; Levstik y Groth, 2005; McKeough et al., 2008; Virta, 2009).

En la escuela colombiana y en los textos escolares durante el siglo xx, por ejemplo, se reprodujeron de forma hegemónica tres mitos fundacionales en las narrativas independentistas que enfatizan más en las intenciones individuales de los sujetos históricos, y menos en el desarrollo de los procesos sociales en los cuales estos sujetos participaban (Múnera, 1998, citado por Vargas, Acosta y Sánchez, 2013). El primer mito es la unidad territorial, política y cultural de la Nueva Granada en el momento de la Independencia, de forma tal que las narrativas reconstruyen los eventos ocurridos en la ciudad de Santa Fe como el epicentro de la planeación y declaración de la Independencia. El segundo mito es que los ideales de la élite criolla sobre una nación independiente permitieron que el pueblo se levantara en contra del Gobierno de España el 20 de julio de 1810. Para finalizar, el tercer mito es que la Independencia de la Nueva Granada fue obra exclusiva de los blancos-criollos, y se les resta agencia a los indígenas, a los afrodescendientes y a las castas. Como resultado, Ios textos escolares y los profesores reproducen en la escuela narrativas históricas acríticas que recuperan como una verdad revelada, por un lado, anécdotas como el préstamo del Florero de Llorente planeado por los criollos para iniciar la reyerta del 20 de julio de 1810; y, por otro, narrativas sobre eventos político-militares que permitieron construir una comunidad moderna occidental, por ejemplo, la campaña libertadora iniciada y dirigida por héroes criollos (Vargas, Acosta y Sánchez, 2013).

En síntesis, la literatura revela que, aunque se encuentre homogeneidad en las narraciones históricas escolares en cada contexto sociocultural particular, también hay espacio para narrativas históricas disímiles en una misma sociedad, las cuales representan creencias particulares del grupo de referencia con el cual se identifican los estudiantes. En este estudio, se pretende conocer cómo se reproduce y se transforma la historia escolar en dos contextos socioculturales disímiles. Además, esto sirve como un proxy, por un lado, para conocer cómo se reconstruye y reproduce la 
historia a través de instrumentos culturales (los textos escolares); y, por otro, para conocer cómo los estudiantes transformaron la historia que es reproducida en los textos escolares.

\section{Método}

\section{Participantes}

Por conveniencia fueron seleccionados y entrevistados 21 estudiantes de octavo grado de secundaria (11 mujeres y 10 hombres), entre los 13 y 14 años $(M=13,12 ; D E=0,32)$, de dos colegios urbanos $(n=11)$ y dos colegios rurales $(n=10)$; el número de entrevistas dependió del criterio de saturación de las categorías investigadas (Small, 2009). Seleccionar estudiantes de octavo grado determinó que ellos habían cursado el tema de la Independencia de Colombia en su currículo escolar, y cognitivamente se encontraban preparados para representar de forma abstracta los procesos sociales del pasado (Barton, 2001).

Los estudiantes fueron seleccionados de dos entornos diferentes (dos colegios rurales de la vereda de Siachoque, Boyacá; y dos colegios urbanos del distrito de Bogotá) con el fin de acceder a formas sustancialmente diversas de enmarcar y situar el proceso de enseñanza y aprendizaje de la historia de la Independencia de Colombia. En los colegios rurales se seleccionaron solo estudiantes indígenas pertenecientes al Cabildo Muisca, ya que la literatura reporta que esta población tuvo agencias diversas en el proceso de Independencia, y se esperaba que este tipo de agencias constituyeran tramas diferenciadas en las narrativas de los estudiantes indígenas, en comparación con los de aulas urbanas.

\section{Diseño y procedimiento}

Esta investigación descriptiva adoptó el análisis de las narrativas para describir y comprender de forma holística cómo las personas interpretan el mundo pasado, sus agentes y el funcionamiento de la sociedad en el pasado, preservando el contexto sociocultural en el que este discurso se ha producido (Bamberg y Cooper, 2011; Riessman, 2002). Previa a la recolección del dato, se solicitó consentimiento informado de todos los participantes, y se guardaron todos los procedimientos legales y éticos para el manejo de los datos recolectados en virtud de la investigación.

La narrativa fue el dato recolectado. En primer lugar, se recogieron las narrativas de los estudiantes a través de una pregunta generativa de narración espontanea: "Cuéntame, ¿cómo ocurrió la Independencia de Colombia?". La pregunta generativa fue construida a partir de entrevistas 
pilotos, y permitió privilegiar la narración espontánea del estudiante sin ninguna intervención directa del entrevistador para reconstruir la narración tal y como se produce. En segundo lugar, se tomaron las narrativas en los capítulos sobre la Independencia de Colombia de los cinco textos de ciencias sociales usados por los estudiantes entrevistados. La decisión de incluir los textos radica en que estos son instrumentos culturales particulares del contexto sociocultural en el que ocurre el aprendizaje. En este estudio se encontró que los textos oficiales para la escuela nueva rural no circulan entre los estudiantes; en todos los casos, los textos escolares que circulan en las aulas fueron producidos por editoriales privadas (Norma, Santillana, Libros \& Libros, SM).

\section{Plan de análisis}

De acuerdo con los resultados de pruebas pilotos previas, se codificó y usó el mismo tipo de análisis para las narrativas de los estudiantes y los textos escolares. En este proceso, se identificaron los temas canónicos y la estructura historiográfica que subyacía a las narrativas para lograr una explicación integrada acerca de cuáles fueron los elementos historiográficos característicos en las producciones narrativas de los textos, cómo se tramaron estos elementos, y cómo los estudiantes transformaron las producciones narrativas de los textos escolares para identificar las comunalidades y las diferencias entre ambas. Para finalizar, el dato narrativo fue comparado entre los colegios rurales y urbanos. A continuación, se describen las tres variables historiográficas rastreadas.

\section{Fuentes históricas}

Mapear las fuentes históricas permite conocer en qué se basó el narrador para construir su conocimiento histórico. El uso de las fuentes se refiere a cómo los recursos históricos primarios y secundarios, considerados más disciplinares, se articulan con las fuentes de la memoria colectiva, consideradas más populares (Plá, 2005). Las fuentes primarias son las fuentes documentales o materiales de primera mano relativas al fenómeno histórico estudiado (monedas, cartas, periódicos, actas, testimonios de protagonistas, congresos) y las fuentes secundarias son una descripción histórica construida a partir de las fuentes primarias en un tiempo diferente a la ocurrencia del evento pasado (textos escolares, un libro). Por su parte, las fuentes de la memoria colectiva son las fuentes informales (no disciplinares) que construyen narraciones históricas (un programa de televisión, narrativas orales en la familia). El uso de las fuentes historiográficas en la historia escolar se opone a una concepción histórica como verdad revelada, en la cual las fuentes no son corroboradas, controvertidas o contrastadas. Por el contrario, la presencia de fuentes en la narrativa transmite que el 
conocimiento histórico se construye como una discusión entre diversas fuentes históricas que son relativizadas a través de la corroboración o contrastación de fuentes provenientes de la memoria colectiva.

\section{La explicación histórica}

Para conocer cómo la historia fue reconstruida como un todo de acontecimientos que ocurrieron en tiempos pasados es necesario conocer las formas de explicación histórica (Burke, 1993; González y González, 1998). La explicación histórica evaluó el papel asignado a la intención dentro de la narrativa. Una narrativa particular puede enfocarse en las intenciones como elementos explicativos dentro de la historia o, por el contrario, en los contextos y los elementos analíticos presentes en estos. Incluso, la narrativa puede enfocarse en la articulación de elementos explicativos y elementos analíticos.

\section{Motor de la historia}

El motor de la historia se refiere a los elementos que permiten que las transformaciones históricas sean posibles en ciertos momentos particulares (Aron, 1984; Veyne, 1984). A diferencia de la explicación histórica que evaluó el enfoque de la narración, el motor de la historia se refiere a los elementos que determinan las transiciones históricas. Puede, por ejemplo, existir una narrativa enfocada en las intenciones de actores particulares (Simón Bolívar), pero que considera que los cambios se producen no por esas intenciones, sino por las luchas sociales existentes (las contradicciones existentes en la Patria Boba y la Restauración Monárquica), o viceversa. Incluso, cada uno de estos motores de la historia puede presentarse de forma exclusiva en la narración.

\section{Resultados}

\section{Fuentes historiográficas como elementos legitimadores}

Los hallazgos evidenciaron que consistentemente los estudiantes, al igual que los textos escolares, no explicitaron fuentes historiográficas en sus narraciones, y por esa razón, reconstruyeron la historia como una verdad revelada. Este patrón fue observado en casi la totalidad de producciones en colegios rurales (Rueda, et al., 2007; Sánchez et al., 2009) y en colegios urbanos (Márquez, 2009; Morelos et al., 2010; Narváez, Ibarra y Bonilla, 2009).

A pesar de este patrón general, excepcionalmente, algunas producciones narrativas de estudiantes y de algunos textos escolares usaron fuentes primarias historiográficas. Sin embargo, las fuentes no fueron incluidas para relativizar 
la historia narrada a través de corroboración, evaluación o contrastación de la evidencia ofrecida en las fuentes, como lo sugieren los historiadores expertos. En su lugar, estas fuentes primarias fueron utilizadas para incluir en la narración la lucha social, política y bélica entre criollos y españoles, así como para enfatizar sobre las intenciones y motivaciones de los criollos por tener los mismos derechos sociales y políticos de los españoles, o por consolidar la Independencia y la unión de naciones.

En ocasiones, los textos escolares incluyeron fuentes primarias oficiales como la Declaración de los Derechos del Hombre y del Ciudadano, Las reformas borbónicas, la Expedición Botánica y el Cabildo Abierto, con el fin de recrear la independencia como una invariante de la constitución de la nueva nación. En otras ocasiones se usaron fuentes documentales privadas tales como el Memorial de Agravios y la Carta de Jamaica para enfatizar en las intenciones independentistas de Simón Bolívar. No obstante, estas fuentes historiográficas fueron usadas por el narrador para incluir acontecimientos históricos memorables en la trama narrativa del proceso de la independencia, y no fueron usadas para relativizar el conocimiento histórico a partir del método histórico (Wineburg, 2001).

Por su parte, aunque los estudiantes no usaron fuentes primarias, un pequeño subgrupo de estudiantes usó fuentes de la memoria colectiva. En estos casos, los estudiantes remplazaron las fuentes históricas, presentes en los textos, por fuentes de la memoria colectiva, pero reprodujeron en sus relatos la misma relación con las fuentes que se observa en los textos. En consecuencia, los estudiantes, al igual que en los textos escolares, no articularon estas fuentes a otras historiográficas primarias o secundarias, ni tampoco relativizaron el hecho de que estas no son una verdad absoluta. Esto es importante, porque involucrar fuentes de la memoria colectiva debería permitir controvertir la narración con otra información no oficial, lo que no sucede. Como resultado, los estudiantes construyeron narraciones históricas desde una mirada acrítica que no relativizó las fuentes, ni revisó los puntos de vista desde los cuales fueron construidas. Por ejemplo, la estudiante 97 de colegio rural refirió un video visto en clase para ofrecer credibilidad a su narración sobre la anécdota del préstamo del Florero de Llorente, pero no relativiza esta fuente histórica:

97: len el video que vimos en clase/ Llorente era un señor [...] ya con sus años (.)/pues no tan viejito/ él era como un señor no tan violento /y eso... qué más... /creo que él era el dueño del florero /y como no se lo prestaron a los hermanos Moreno/ entonces él creía que los hermanos Moreno no le iban a devolver el florero/ entonces él no se los prestó (.) /que comenzaron a tratarlo mal/ así(.) lo empezaron a tratar mal:: / púes que eso/ digamos [...] eso estaba en el pasado /y eso digamos eso es como de sociales /y digamos tiene que ver... tiene que ser algo relacionado con la Independencia// 
En suma, el uso de fuentes históricas en las narraciones reproducidas por los textos escolares puso a disposición de los estudiantes formas poco complejas de reconstruir el conocimiento histórico escolar y, en esta misma línea, usaron esta forma poco compleja para construir su propio conocimiento.

\section{Explicar la historia desde las intenciones o el contexto histórico}

Aunque en la mayoría de los casos la Campaña Libertadora regularmente fue explicada a través de las intenciones de los héroes o líderes criollos, los hallazgos evidenciaron que en un subgrupo de textos (Morelos et al., 2010; Sánchez et al., 2009) se articularon elementos explicativos de la estructura de la sociedad (ideas de la llustración, restricciones económicas y de comercio en las colonias americanas, principios de la Revolución francesa) con las intenciones y expectativas de los criollos quienes esperaban una oportunidad para separarse de España.

En estos casos, tramar cómo funcionó la estructura de la sociedad permitió contextualizar cómo fue posible que los criollos idearan movimientos que depusieron a las autoridades españolas en América, para luego instalar sus propias juntas de gobierno. Desde esta versión, es posible que "la invasión de España en 1808 por parte de Francia" haya facilitado el surgimiento de los movimientos independentistas: "La noticia despertó en algunos criollos interés, porque desde 1808 estaban aguardando una oportunidad para separarse de España" (Morelos et al., 2010; pp. 90-91). No obstante, estas narraciones omitieron la articulación detallada de la forma en la que las intenciones se relacionaban con los elementos analíticos del contexto, y, en algún nivel, sobredimensionaban el rol de las intenciones en la explicación del cambio histórico. Este patrón fue observado en casi la totalidad de producciones en colegios rurales y en colegios urbanos.

Un panorama similar se encontró en las narraciones de los estudiantes de colegios rurales y urbanos que, en su mayoría, se concentraron en las intenciones $y$, cuando incluyeron factores del contexto, lo hicieron privilegiando la intención individual sobre los factores contextuales. Analicemos algunos fragmentos de la narración principal del estudiante 71 de colegio urbano, quien, si bien privilegió las intenciones individuales, también incluyó elementos del contexto, pero ambos de forma desarticulada. El estudiante construyó una narración principal en la que constantemente se introdujeron personajes históricos, por ejemplo,

Antonio Zea o Antonio Nariño (.) entre otros (.)/ planearon ir a prestar un florero al Señor Llorente... comenzaran a armar las revoluciones dirigidas por el señor Simón Bolívar... grandes generales como fue 
Antonio Nariño (.) Francisco de Paula Santander, entre muchos otros... grandes como Policarpa Salabarrieta o Manuela Beltrán tampoco se les hizo fácil. (Estudiante 71).

El estudiante 71, de forma transversal en su narración privilegió las intenciones de los sujetos históricos sobre otros elementos relevantes en el proceso de la independencia. Por ejemplo:

Las personas que iniciaron como esa revolución también o la insurrección de los comuneros /que fueron hechos que al final llevaron a ese grito de independencia(.) /lo que llevó al cabildo abierto (.) /lo que llevó a que en la Nueva Granada hubiera más leyes /que al final de todo Bolívar logró derrotar / ... y al final. Yo digo que fue la fuerza de la gente lo que logró como esa independencia.

Sin embargo, en algunos momentos de la narración principal, el estudiante identificó las intenciones de los sujetos históricos colectivos (por ejemplo, criollos) y las articuló no solo las intenciones de sujetos individuales (por ejemplo, Simón Bolívar), sino con situaciones más contextuales o causas materiales, complejizando así el nivel de la narración. Por ejemplo:

[...] entonces, aparte de eso, surgió el cabildo abierto/ que fue muchas conversaciones/ en las que se planteó que los criollos querían más participación (.)/ que no todo tuviera que ver con solo los españoles/ y como que hubiera un poquito más de independencia/// digamos fue como la apertura/ para que se comenzaran a armar las revoluciones dirigidas por el señor Simón Bolívar/ que entre muchas otras cosas tuvo relaciones/ y pidió ayuda varias veces (i?)/ y se dice que hubo mucha dependencia extrajera/ sobre todo de Inglaterra/ en el sentido de que ni Venezuela ni la Nueva Granada estaban en la capacidad de sostener una guerra/ pero se supone que Inglaterra fue un gran [...] fue uno de los países que más apoyó la Independencia en Colombia. (Estudiante 71).

En suma, la narración del estudiante 71 logra tipificar tres momentos en las formas de transformación de las narraciones de los textos escolares. En ocasiones, se revela un conocimiento más concreto de las instituciones y realidades sociales, considerando solo los sujetos históricos individuales que encarnan diferentes roles institucionales. En otros momentos, el estudiante articuló las intenciones individuales y colectivas, o, por el contrario, privilegió las acciones colectivas, reconociendo el impacto que estas poseen sobre la ocurrencia de eventos y desmitificando a algunos héroes o personajes protagónicos de la historia. Sin embargo, la producción narrativa en su conjunto aún no evidencia una comprensión histórica compleja que dé cuenta de cómo los diferentes elementos explicativos de la narración histórica se interconectan y se definen dinámicamente por su relación con otros aspectos de esa historia particular. 
En síntesis, el foco principal de la narración histórica fueron las intenciones individuales. Aunque en ocasiones se articula el uso de condiciones del contexto, este uso solo se revela como una vía para ubicar la ocurrencia del evento con escasas descripciones. En consecuencia, las pocas descripciones en las narraciones y el uso de elementos explicativos, basados más en las intenciones de los sujetos, no logran revelar comprensiones históricas complejas en los estudiantes, ni en los textos escolares.

\section{Narrar el motor de la historia desde las ideas o desde las causas materiales}

La reconstrucción de los cambios y las transformaciones históricas dentro de las producciones narrativas, de estudiantes y los textos escolares en contextos rurales y urbanos, en su mayoría se refirieron a las intenciones como el motor que movió el cambio en el devenir histórico. Esto es, la mayoría de las narraciones privilegiaron las ideas de los sujetos protagonistas de la historia como el motor del cambio. Por ejemplo, en los textos escolares de ambos tipos de colegios, se narró la Patria Boba como un periodo de la historia, en el cual primaron las ideas de los sujetos individuales y colectivos como punto de inflexión del cambio; esto es, las pugnas ideológicas entre los seguidores de Antonio Nariño y los seguidores de Camilo Torres.

En esta misma línea, los textos escolares usados en los colegios rurales y urbanos se entramaron las ideas de la élite criolla como el motor que movió la Campaña Libertadora. En la narración histórica de este evento se reconstruyó cómo Simón Bolívar y Francisco de Paula Santander cobraron el protagonismo de la élite criolla, porque ellos pensaron cómo lograr la Independencia definitiva de las provincias en América, después del proceso de reconquista española entre 1815 y 1819.

Similarmente, la mayoría de estudiantes privilegiaron las ideas de los sujetos históricos, especialmente, para narrar el inicio de la Independencia y la Campaña Libertadora. En muy pocas ocasiones incluyeron en sus narraciones la Patria Boba o la Reconquista como puntos de inflexión. En particular, los estudiantes incluyeron la negativa del préstamo del florero por parte de José Llorente como lo que generó la Independencia o la Batalla de Boyacá, y también enfatizaron en el malestar de la élite criolla por el maltrato al que eran sometidos diferentes grupos de pobladores de América (los esclavos, los criollos, los indígenas) como aquello que ocasionó el cambio histórico de la Colonia a la República. Por ejemplo, el estudiante 6 de un colegio urbano narró las ideas de los criollos sobre la participación en el gobierno como el centro del cambio de la Colonia al inicio de Independencia: 


\begin{abstract}
[...] /pues digamos que fue como un plan/ para abrir todo el proceso/// y entonces aparte de eso surgió el cabildo abierto/ que fue muchas conversaciones/ en las que se planteó que los criollos querían más participación (.)/ que no todo tuviera que ver con solo los españoles/ y como que hubiera un poquito más de independencia/// digamos fue como la apertura/ para que se comenzaran a armar las revoluciones dirigidas por el señor Simón Bolívar. (Estudiante 6).
\end{abstract}

En síntesis, el estudiante 6, al igual que otros de octavo grado de colegios urbanos y rurales, narró el plan criollo de la revuelta como lo que desencadenó los cambios en la historia. En estas narraciones, se destacó la primacía de los criollos como los pensadores del plan de Independencia. Simón Bolívar dentro del relato fue construido como un líder memorable del proceso, quien fue incluido en el proceso de reconquista española, Campaña Libertadora y conformación de la República. Como resultado, se resta la agencia que otros colectivos que participaron el plan de la revuelta del 20 de julio de 1810 (por ejemplo, esclavos, campesinos, indígenas) u otros líderes criollos (por ejemplo, Antonio Nariño, Camilo Torres).

\title{
Discusión
}

Los hallazgos revelaron que, en los contextos escolares analizados, se reprodujo una narrativa histórica nacional que estructuralmente no favorece la construcción de conocimiento disciplinar sofisticado, en la medida en que se enfatizó más en descripciones de acontecimientos y sujetos históricos memorables, y menos en justificar o explicar el proceso de Independencia. Adicionalmente, estas narrativas guardaron una estrecha relación con la construcción narrativa de la identidad nacional de los estudiantes, de forma tal que se perpetúan anécdotas (por ejemplo, anécdota del préstamo del florero de Llorente) y algunos mitos fundacionales (por ejemplo, enfatizar en las intenciones de los criollos como la única razón para que el pueblo se levantará en contra del Gobierno de España, o atribuir a ideas de los blancos-criollos la realización de la Campaña Libertadora). Este tipo de reproducción histórica se alejó de posturas críticas y reflexivas para la reconstrucción del conocimiento histórico.

Las comunalidades entre las producciones narrativas de los estudiantes y sus textos escolares suponen que estos últimos presentan una plantilla narrativa que, en alguna medida, es interiorizada por los estudiantes, y les permite luego producir narrativas históricas por sí solos (Cole, 1996). La plantilla reproducida en los textos para organizar la información y otorgarle significación histórica constituyó en sí una herramienta cultural. En términos generales, estudiantes y textos reconocieron la narrativa nacional oficial que incluyó: a) similares sujetos históricos memorables (Llorente, 
los hermanos Morales, Simón Bolívar); b) eventos similares (préstamo del florero el 20 de julio de 1810, Batalla de Boyacá el 7 agosto de 1819); c) punto de inflexión similar (cansancio de los criollos por la desigualdad política, económica y social entre colonizados y colonizadores, así como la lucha bélica por la igualdad), y d) una solución similar (libertad).

En esta línea, consistentemente, los textos reprodujeron con más frecuencia las intenciones de los sujetos individuales para explicar la ocurrencia de los eventos históricos y las ideas de estos sujetos como el motor de la historia. En consecuencia, los estudiantes privilegiaron el uso de indicadores del menor nivel de sofisticación, como enfatizar en las ideas y las intenciones para reconstruir la historia de la Independencia.

Este tipo de reproducciones narrativas independentistas va en contravía de lo dispuesto por el Ministerio de Educación Nacional (MEN) frente a la reconstrucción de las narrativas oficiales de la Independencia en el marco del Bicentenario, momento en el cual se publicó el texto escolar oficial para enseñar y aprender la Independencia de Colombia (MEN, 2010). En esta reconstrucción narrativa se enfatiza en los elementos analíticos de la historia (causas materiales, circunstancias coyunturales, luchas sociales) como la forma de favorecer la construcción de conocimiento histórico, que más cercano al modelo experto, posibilite la representación abstracta de los procesos sociales vinculados al proceso de independencia. Sin embargo, en esta investigación se encontró que los textos que circulan en los contextos escolares analizados no solo se alejan de las narrativas independentistas oficiales, sino que, además, los textos escolares oficiales del MEN no circulan dentro de los colegios oficiales participantes.

La no circulación de los textos escolares producidos por el Estado, tal vez, no permitió encontrar diferentes tipos de narrativas en los contextos rurales y urbanos. Además, el alejamiento de las narrativas históricas reproducidas en los textos escolares de editoriales privadas, que son los más usados en los colegios, revela que no se tienen en cuenta los elementos analíticos dispuestos por el MEN, y que en su lugar se continúan privilegiando los elementos narrativos vinculados a mitos fundaciones, que según lo revela la investigación histórica han prevalecido en la enseñanza de la historia en la escuela desde el siglo xx. En esta línea, sería necesario generar políticas estatales que favorezcan la revisión de los lineamientos a la hora de construir textos escolares y formas de revisión de pares expertos del Estado que verifiquen el cumplimiento de lineamientos en la construcción disciplinar, como se hacen en otros países (México, Estados Unidos).

Por otra parte, en esta investigación se encontró que coexisten las mismas narrativas independentistas en contextos rurales y urbanos. Aunque esto no debería ser problemático en principio, el problema radica es que los estudiantes rurales siendo indígenas no recuperan discursos sociales 
propios de su identidad social. Este elemento requiere estudios futuros, sin embargo, nos atrevemos a inferir que las narrativas históricas que no recuperan la agencia campesina o indígena en la construcción de narrativas independentistas, ni la significancia social y económica de los grupos indígenas generaron la independencia, no posibilitan en los estudiantes recursos identitarios importantes en los procesos de identificación social indígena.

En este sentido, los editores de los textos escolares deberían realizar un esfuerzo consciente por recuperar aquellos elementos de la memoria histórica y de las fuentes primarias y secundarias relevantes para la reconstrucción identitaria de los grupos sociales indígenas (de América para octavo grado: mexicanos, peruanos, gauchos). Este tipo de elementos, entramados de forma compleja, posibilitarían, además, la comprensión de relaciones temporales y causales entre las gestas criollas y las gestas indígenas, u otros grupos sociales participantes.

\section{Referencias}

Acevedo, A. y Samacá, G. (2012). La política educativa para la enseñanza de la historia de Colombia (1948-1990): de los planes de estudio por asignaturas a la integración de las ciencias sociales. Revista Colombiana de Educación, 62, 221-244.

Alridge, D. (2006). The limits of master narratives in history textbooks: An analysis of representations of Martin Luther King, Jr. The Teachers College Record, 108(4), 662-686. Recuperado de http:// civilrightsteaching.org/wp-content/uploads/2013/08/Alridge-Essay-on-King-and-textbooks.pdf.

Aron, R. (1984). Introducción a la filosofía de la historia. Ensayo sobre los límites de la objetividad histórica completado con textos recientes. Vol. I. Buenos Aires: Siglo Veinte.

Ashby, R. (2004). Developing a concept of historical evidence. Students' ideas about testing singular factual claims. International Journal of Historical Learning, Teaching and Research, 4(2), 44-55. Doi: http:// www.ex.ac.uk/historyresource/journalstart.htm.

Bamberg, M. y Cooper, I.H. (2011). Narrative analysis. APA handbook of research methods in psychology. Vol. 3. Washington, DC: APA Press.

Barton, K.C. (2001). A sociocultural perspective on children's understanding of historical change: Comparative findings from Northern Ireland and the United States. American Educational Research Journal, 38(4), 881-913. Doi: 10.3102/00028312038004881. 
Barton, K.C. y McCully, A.W. (2005). History, identity, and the school curriculum in Northern Ireland: an empirical study of secondary students' ideas and perspectives. Journal of Curriculum Studies, 37(1), 85-116. Doi:10.1080/0022027032000266070.

Burke, P. (1993). Historia de los acontecimientos y el renacimiento de la narración. En P. Burke (ed.), Formas de hacer historia (pp. 287-306). Madrid: Alianza.

Cardoso, N. (2001). Los textos de lectura en Colombia. Aproximación histórica e ideológica. 1872-1917. Revista Educación y Pedagogía, XIII(29-30), 131-142.

Carretero, M.; Castorina, J.A. y Levinas, L. (2011). Conceptual change and historical narratives about the nation. A theoretical and empirical approach. En International Handbook of Research on Conceptual Change (pp. 1-18). 2a. ed. Nueva York, Routledge.

Cole, M. (1996). Cultural psychology: A once and future discipline. Cambridge: Harvard University Press.

Dan, Y.; Todd, R. y Lan, W. (2010). Consensus and Difference: American Students' Perspectives on the National History. Education, 131(2), 331-341.

Epstein, T. (2006). The effects of family/community and school discourses on children's and adolescents' interpretations on United States history. International Journal of Historical Learning, Teaching and Research, 6(1), 1-9. Recuperado de https://bit.ly/2LMnyod

Fuchs, E. (2011). Current trends in history and social studies textbook research. Journal of International Cooperation in Education, 14(2), 17-34.

González y González, L. (1998). El oficio del historiar. México: Clío.

Lee, P. (2005). Putting principles into practice: Understanding history. En M.S. Donovan y J.D. Bransford (eds.), How students learn: History in the classroom (pp. 31-78). Washington: National Academies Press.

Levstik, L.S. (2008). What happens in social studies classrooms? En L.S. Levstik, L. S. y C.A. Tyson (eds.), Handbook of Research on Social Studies Education (pp. 50-64, cap. 4). Reino Unido: Taylor \& Francis.

Levstik, L.S. y Barton, K.C. (2010). Doing history: Investigating with children in elementary and middle schools. Reino Unido: Taylor \& Francis.

Levstik, L. y Groth, J. (2005). Ruled by Our Own People: Ghanaian Adolescents' Conceptions of Citizenship. The Teachers College Record, 107(4), 563-586.

Márquez, C. (ed.) (2009). Sociales: Relaciones ciencias Sociales 8. Bogotá: Libros \& Libros S.A. 
McKeough, A.; Bird, S.; Tourigny, E.; Romaine, A.; Graham, S.; Ottmann, J. y Jeary, J. (2008). Storytelling as a foundation to literacy development for Aboriginal children: Culturally and developmentally appropriate practices. Canadian Psychology/Psychologie Canadienne, 49(2), 148. doi: 10.1037/0708-5591.49.2.148.

Ministerio de Educación Nacional (MEN) (2010). Historia hoy: aprendiendo con el bicentenario. Recuperado de: http://www.colombiaaprende.edu.co/html/productos/1685/article-200229.html

Morelos, L. et al. (2010). Retos sociales 8. Bogotá: Editorial Norma.

Narváez, G.; Ibarra, A. y Bonilla, M. (2009). Viajeros. Sociales 8. Bogotá: Norma.

Plá, S. (2005). Aprender a pensar historicamente. La escritura de la historia en el bachillerato. Barcelona: Plaza y Valdés. S.A. de C.V.

Repoussi, M. y Tutiaux-Guillon, N. (2010). New trends in history textbook research: issues and methodologies toward a school historiography. Journal of Educational Media, Memory, and Society, 2(1), 154-170. Doi: http://dx.doi.org/10.3167/jemms.2010.020109.

Riessman, C.K. (2002). Narrative analysis. En A.M. Huberman y M.B. Miles (eds.), The qualitative researcher's companion (pp. 217-270). Londres: Sage.

Rodríguez, J. (2004). Rutas pedagógicas de la historia en la educación básica de Bogotá. Bogotá: Universidad Nacional de Colombia, Programa RED e Instituto de Investigación Educativa y Desarrollo Pedagógico (IDEP).

Rodríguez, S. (2010). Construcción de la memoria oficial en el Centenario de la Independencia: el Compendio de Historia de Colombia de Henao y Arrubla. Folios, 32, 23-42.

Rueda, P. et al. (2007). Ciencias Sociales 8. Bogotá: Santillana

Samacá, G.A. (2010). Manuales escolares de Ciencias Sociales y proyecto de memoria nacional a principios de la década del noventa del siglo xx: Colombia, una república democrática y en vías de desarroIlo. Revista Anuario de Historia Regional y de las Fronteras, 15(1), 206-223.

Sánchez, G. et al. (2009). Viajeros sociales 8. Bogotá: Editorial Norma.

Small, M.L. (2009). How many cases do I need? On science and the logic of case selection in field-based research. Ethnography, 10(1), 5-38. Doi: 10.1177/1466138108099586.

VanSledright, B. (2008). Narratives of nation-state, historical knowledge, and school history education. Review of Research in Education, 32(1), 109-146. Doi: 10.3102/0091732X07311065. 
Vargas, S.; Acosta, M. y Sánchez R. (2013). Historia, memoria, pedagogía: Una propuesta alternativa de enseñanza/aprendizaje de la historia. Bogotá: Corporación Universitaria Minuto de Dios.

Veyne, P. (1984). Cómo se escribe la historia. Foucault revoluciona la historia. Madrid: Alianza.

Virta, A. (2009). Learning to teach history in culturally diverse classrooms. Intercultural Education, 20(4), 285-297. Doi: 10.1080/14675980903351920.

Wertsch, J.V. (1999). Revising Russian History. Written Communication, 16, 267-295. Doi: 10.1177/074108839901600300.

Wertsch, J. V. (2005). Cole's Cross-Cultural and Historical Perspectives on the Developmental consequences of Education. Human Development, 48(4), 223- 226. Doi: 10.1159/000086857.

Wineburg, S. (2001). Historical Thinking. Filadelfia: Temple University Press. 
